\title{
Impact of Abdominal Obesity on Thyroid Auto-Antibody Positivity: Abdominal Obesity Can Enhance the Risk of Thyroid Autoimmunity in Men
}

\author{
Jazyra Zynat $\mathbb{D}$, Suli Li, Yanrong Ma, Li Han, Fuhui Ma, Yuyuan Zhang, Bei Xing, \\ Xinling Wang, and Yanying Guo \\ Department of Endocrinology, People's Hospital of Xinjiang Uygur Autonomous Region, Ürümqi, Xinjiang, China \\ Correspondence should be addressed to Yanying Guo; guozeyang@126.com
}

Received 5 September 2019; Revised 29 October 2019; Accepted 6 December 2019; Published 13 March 2020

Academic Editor: Flavia Magri

Copyright ( $\odot 2020$ Jazyra Zynat et al. This is an open access article distributed under the Creative Commons Attribution License, which permits unrestricted use, distribution, and reproduction in any medium, provided the original work is properly cited.

Background. The interrelation between obesity and autoimmune thyroid diseases is complex and has not been confirmed. The aim of the present study was to observe the relationship between thyroid autoimmunity and obesity, especially abdominal obesity, in a large population. Methods. A total of 2253 residents who had lived in Xinjiang for more than 3 years were enrolled. Serum thyroid hormone concentration, thyroid autoantibodies, lipid parameters, Weight, height, and waist and hip circumference were measured. Results. The prevalence of thyroid peroxidase antibody (TPOAb) and/or thyroglobulin antibody (TgAb) positive was $32.1 \%$ (21.2\% in men and $37 \%$ in women, $P<0.01)$. Compared with women, men had significantly higher TG levels, waist circumference, and hip circumference levels $(P<0.01)$, while women showed higher TSH, TPOAb, and TgAb levels $(P<0.01)$. The prevalence of overweight and obesity was $71.1 \%$ in men and $63.5 \%$ in women. Men had a higher prevalence of abdominal obesity than women $(56.6 \%$ in men and $47.6 \%$ in women, $P<0.01)$. TPOAb correlates positively with waist circumference $(r=0.100, P<0.05)$ in men. Binary logistic analysis showed that TPOAb positivity had increased risks of abdominal obesity in men, and the OR was 1.1044 (95\% CI 1.035, 1.151, $P<0.05)$. Conclusion. Our results indicate that men had higher lipid levels, thicker waist circumference, and higher prevalence of overweight, obesity, and abdominal obesity. Abdominal obesity is a risk factor for TPOAb positivity in men, suggesting that abdominal obesity can enhance the risk of thyroid autoimmunity in men.

\section{Introduction}

Autoimmune thyroid diseases (AITDs) are the most common autoimmune diseases in humans; one of these is Hashimoto's thyroiditis (HT). HT is a common organspecific autoimmune disorder, which presents the lymphocytic infiltration of the thyroid gland and the production of autoantibodies [1]. The major autoantigens in Hashimoto's disease are thyroid peroxidase (TPO) and thyroglobulin $(\mathrm{Tg})$ antibodies, and the annual incidence of HT worldwide is estimated to be 0.3 to 1.5 cases per 1000 persons [2]. Intrathyroidal lymphocytic infiltration may lead to subclinical or overt hypothyroidism [3]; meanwhile, subclinical or overt hypothyroidism is an important risk factor for cardiovascular diseases [4]. Many studies reported that hypothyroidism causes a number of health issues, including insulin resistance, dyslipidemia, central adiposity, obesity, and chronic inflammation and further contributes to the development of atherosclerosis $[5,6]$. Notably, recent researches have shown that even autoimmune thyroid disease patients in euthyroid still have more early atherosclerotic lesions $[7,8]$.

Obesity, like thyroid diseases, is a common disorder in the general population and it often occurs in a single individuals. A lot of studies showed that obesity is an important risk factor for many diseases, such as coronary heart disease, stroke, diabetes, and some types of cancer [9-11]. Thyroid dysfunctions, such as hypothyroidism, have been extensively investigated in obese subjects. Some studies have found that obesity increases the risk of hypothyroidism 
$[12,13]$. Studies demonstrated that thyroid stimulating hormone (TSH) is positively correlated with body mass index (BMI), and they propose the positive rate of antibody in obese patients with subclinical hypothyroidism is also lower than that in nonobese patients [14]. While it is well known that hyperthyroidism leads to weight loss and hypothyroidism is associated with weight gain; the changes of thyroid function are discussed controversially in obesity [15]. Recent studies have shown that obesity may increase the risk of autoimmune diseases such as inflammatory bowel disease, psoriatic arthritis, and rheumatoid arthritis, suggesting a possible link between obesity and autoimmunity $[16,17]$. Another study suggests that visceral adipose tissue (VAT) is a key component of the body's immune system. Immune cells affect the metabolism of adipocytes. In turn, adipocytes regulate the function of immune cells and provide energy for their activities. In addition, adipocytes themselves produce antibacterial peptides, proinflammatory cytokines, and adipokines. These substances work together to fight infections, alter the function of immune cells, and maintain metabolic balance [18]. Therefore, there may exist a possible common pathway between thyroid autoimmunity and abdominal obesity, especially with visceral adipose tissue in abdominal obesity subjects.

We hypothesize that the abdominal obesity may play a potential role in the development of autoimmune thyroid diseases. Although some observational studies have evaluated the effects of obesity on thyroid dysfunction, the relationship between abdominal obesity and autoimmune thyroid diseases remains undetermined. The data used in this study were from the Survey on Prevalence in Thyroid Diseases and Risk Factors in Xinjiang, China. The aim of the study was to define the influence of abdominal obesity on Hashimoto's thyroiditis among Xinjiang Chinese Natural population.

\section{Materials and Methods}

2.1. Study Participants. The study was approved by the ethics committee of the People's Hospital of Xinjiang Uyghur Autonomous Region, and all participants provided written informed consent before data collection. This is a population-based cross-sectional study and conducted in two communities of Urumqi in Xinjiang Province, during May-July, 2013. Adults aged 16 years or older who were Chinese citizens and had lived in their current residence were invited to participate in our study. The exclusion criteria comprised subjects with severe communication problems, had received thyroid therapy including medicine and/or surgery or radiotherapy and severe/acute illness, or who were unwilling to participate.

A total of 2253 subjects enrolled initially. Participants with missing laboratory results $(n=193)$, anthropometric measurement data $(n=137)$, and questionnaire data $(n=35)$ were excluded from the study. Finally, 1888 subjects were included in the final analysis (Supplementary Figure 1).

2.2. Data Collection. All data collections were performed by the same staff group from the Department of Endocrinology in People's Hospital of Xinjiang Uyghur Autonomous Region. All staff were trained according to a standard protocol that made them familiar with the specific tools and methods used. A standard questionnaire of each participant was administered by the trained staff group, including demographic characteristics, medical history, previous diagnoses of thyroid diseases, and whether the subject had undergone thyroid therapy, including medicine and/or surgery or radiotherapy for the head and neck. Weight, height, and waist and hip circumference were measured according to a standard protocol. All anthropometric measurements were conducted at the same time when the serum samples were collected.

2.3. Laboratorial Assays. Serum samples for laboratorial assays were drawn after an overnight fast of at least 8 hours. Blood samples were stored at $-20^{\circ} \mathrm{C}$ when collected and shipped to our laboratory within 2-4 hours of collection.

Thyroid parameters, serum thyroid-stimulating hormone (TSH), and levels of thyroid peroxidase antibody (TPOAb) and thyroglobulin antibody (TgAb) were measured by a Roche electrochemiluminometric analyzer (Cobas-e601 analyzer, Roche, Mannheim, Germany). Lowdensity lipoprotein (LDL), high-density lipoprotein (HDL), triglycerides (TG), and total cholesterol (TC) were measured by LABOSPECT 008 AS.

2.4. Definition of Variables. The normal reference range for TSH is $0.27-4.2 \mathrm{mIU} / \mathrm{L}$; for TPOAb, it is $0-35 \mathrm{IU} / \mathrm{mL}$; and for $\mathrm{TgAb}$, it is $0-116 \mathrm{IU} / \mathrm{mL}$ [19].

HT was defined as the serum TPOAb $(>35 \mathrm{IU} / \mathrm{mL})$ and/ or $\mathrm{TgAb}(>116 \mathrm{IU} / \mathrm{mL})$ positivity (TPO/TgAb $(+))$ (Roche).

Normal weight is defined as $18.5 \leq \mathrm{BMI}<24 \mathrm{~kg} / \mathrm{m}^{2}$, while overweight is defined as BMI $\geq 24 \mathrm{~kg} / \mathrm{m}^{2}$, and obese is defined as BMI $\geq 28 \mathrm{~kg} / \mathrm{m}^{2}$ according to the criteria [20].

Central obesity was defined as waist circumference $\geq 85 \mathrm{~cm}$ in females and $\geq 90 \mathrm{~cm}$ in males [20].

Total cholesterol $<5.2 \mathrm{mmol} / \mathrm{L}$, triglycerides $<1.7 \mathrm{mmol} / \mathrm{L}$, LDL-C $<3.4 \mathrm{mmol} / \mathrm{L}$, and HDL-C $>1.0 \mathrm{mmol} / \mathrm{L}$ are considered to be the appropriate level of blood lipids according to Guidelines for the Prevention and treatment of dyslipidemia in Chinese Adults [21].

2.5. Statistical Analysis. We performed survey analyses with IBM SPSS Statistics, Version 20.0 (SPSS, Chicago, IL, USA). A $P$ value $<0.05$ was considered significant. Continuous variables with normal distribution were presented as mean with standard deviation (SD), and categorical variables were presented as the median with the 25 th and 75 th percentiles. Continuous variables were compared using Student's $t$ test. The Mann-Whitney $U$ test was used for nonnormally distributed continuous variables. Correlations of TPO/TgAb positivity with metabolic and lipid parameters were analyzed using Spearman's correlation analysis. The associations among TPO/TgAb positivity and with metabolic and lipid parameters were assessed by logistic regression. Results were expressed as odds ratios ( $95 \%$ confidence interval). The body 
mass index (BMI) was calculated as weight in kilograms divided by height in meters squared.

\section{Results}

3.1. General Characteristics of the Study Population. A total of 1888 subjects were enrolled in this study. The mean age was $46.27 \pm 14.55$ (range, 16-84) years for all subjects, $46.04 \pm 16.41$ (range, $16-83$ ) years in males and $46.37 \pm 13.64$ (range, 17-84) years in females. The mean body mass index (BMI) was $25.96 \pm 4.32 \mathrm{~kg} / \mathrm{m}^{2}$, waist circumference was $85.97 \pm 12.11 \mathrm{~cm}$, and hip circumference was $97.07 \pm$ $8.28 \mathrm{~cm}$. Among them, 585 (31\%) were males and 1303 (69\%) were females. There were evident differences in TSH, TPOAb, TgAb, HDL, TG, waist circumference, and hip circumference levels between men and women $(P<0.01)$.

Men had worse lipid profile compared with women: they had significantly lower HDL cholesterol levels, $1.25 \pm 0.27$ versus $1.48 \pm 0.48 \mathrm{mmol} / \mathrm{L}(P<0.01)$, and higher TG levels, $1.54(0.99-2.14)$ versus $1.16(0.85-1.64) \mathrm{mmol} / \mathrm{L}(P<0.01)$. There was no significant difference in TC and LDL cholesterol levels between the two gender groups $(P>0.05)$.

Thyroid parameters between subjects in men and women also varied significantly. Women subjects showed higher TSH levels (2.66 (1.68-4.10) versus $2.06 \quad(1.45-3.16) \mathrm{uIU} / \mathrm{ml}$, $P<0.01), \quad$ TPOAb $(27.77 \quad(22.61-38.48) \quad$ versus 24.57 (19.60-31.72) IU/ml, $P<0.01)$, and TgAb (31.13 (26.33-93.67) versus $27.53(24.50-32.05) \mathrm{IU} / \mathrm{ml}, P<0.01)$.

When metabolic parameters were evaluated, men had higher waist circumference $(90.24 \pm 11.84$ versus $84.06 \pm$ $11.73 \mathrm{~cm}, P<0.01)$ and hip circumference levels $(97.83 \pm$ 7.57 versus $96.72 \pm 8.55 \mathrm{~cm}, P<0.01)$ than women subjects. However, there were no significant differences in BMI between the two gender groups $(26.14 \pm 3.75$ versus $25.88 \pm$ $\left.4.55 \mathrm{~kg} / \mathrm{m}^{2}, P>0.05\right)$.

Characteristics of the study population, their anthropometric data, metabolic parameters, and thyroid parameters are shown in Supplementary Table 1.

3.2. Clinical Characteristics according to TPOAb and $\operatorname{TgAb}$ Levels. The prevalence of TPOAb and/or $\mathrm{TgAb}$ positive (TPO/TgAb $(+)$ ) was $32.1 \%(21.2 \%$ in men and $37 \%$ in women, $P<0.01$; Supplementary Table 2$)$. The prevalence of overweight and obesity was higher in men $(71.1 \%)$ than women $(63.5 \%)$ in this population $(P<0.01$; Supplementary Table 2). Men had a higher prevalence of abdominal obesity than women $(56.6 \%$ in men and $47.6 \%$ in women, $P<0.01$ ) (Supplementary Table 2).

The characteristics of the participants in terms of TPO/ TgAb positivity are summarized in Supplementary Table 3.

For men, participants with HT had a significantly higher level of waist circumference compared with participants in the TPOAb and TgAb-negative (TPO and $\mathrm{TgAb}(-)$ ) group $(P<0.05)$. BMI was slightly higher in the HT group but did not reach statistical significance $(26.51 \pm 4.12$ versus $\left.26.04 \pm 3.64 \mathrm{~kg} / \mathrm{m}^{2}, P>0.05\right)$. There was no significant difference in hip circumference, TC, HDL, and LDL cholesterol levels between the two groups $(P>0.05)$.
For women, no significant difference was found between the HT (+) and HT (-) groups with regard to BMI, waist circumference, hip circumference, TC, HDL, and LDL cholesterol levels $(P>0.05)$.

3.3. Association of TPO/TgAb Positivity with Metabolic and Lipid Parameters. The Spearman's correlation analysis showed a correlation between $\mathrm{TPO} / \mathrm{TgAb}$ positivity with metabolic and lipid parameters. It was found that TPOAb correlates positively with waist circumference $(r=0.100$, $P<0.05)$, TC $(r=0.051, P<0.05)$ in men, while TPOAb correlates positively with hip circumference $(r=0.084$, $P<0.01)$ and TC $(r=0.081, P<0.05)$ in women (Supplementary Table 4). Given that the findings of TPO positivity were positively associated with metabolic parameters, we evaluated the adjusted odds ratios (ORs) for metabolic in the TPOAb (+) group. Adjusted ORs were calculated after adjusting for age and TSH using the binary logistic regression model. As shown in Supplementary Figure 2, TPOAb positivity was associated with an increased risk of abdominal obesity in men, and the OR was 1.104 (95\% CI $1.035,1.151, P<0.05)$. General obesity and hyperlipidaemia (hypercholesterolemia, hypertriglyceridemia, hyper-low-density lipoproteinemia) had no significant relationship with TPOAb positivity in men subjects $(P<0.05)$. No significant association of TPOAb/TgAb positivity with obesity, abdominal obesity, and hyperlipidaemia was found in women subjects $(P<0.05)$.

\section{Discussion}

In our study, the prevalence of HT was $32.1 \%$ (TPOAb positivity $25.7 \%$, $\mathrm{TgAb}$ positivity $18.6 \%$ ). Compared with the previous epidemiological investigation of thyroid diseases conducted by Teng et al. [22] in 10 cities in China (the prevalence positive TPOAb $11.5 \%$, positive $\operatorname{TgAb} 12.6 \%$ ), this study showed that the positive rate of thyroid autoantibodies in Xinjiang was higher. Our study showed that nearly two-thirds of participants are either overweight (37\%) or obese $(28.9 \%)$, and more than half of the participants are abdominal obese (50.4\%) and are higher than the data from the 2009 China Health and Nutrition Survey (obese 26.4\%; abdominal obese 37.4\%) [23].

The susceptibility to autoimmune thyroid disease depends mainly on the genetic determinants of HLA and nonHLA loci (CTLA4, CD40, PTPN22, TG, and TSH-R genes), and these genetic determinants may be affected by various environmental factors, such as iodine deficiency, stress, drugs, chemical contaminants, and infectious organisms [24]. The prevalence of HT is $21.2 \%$ in men and $37 \%$ in women in our study. Consistent with previous researches [3], the positive thyroid autoantibodies showed substantial correlation with female gender in this research. TPOAb was identified as a major autoantigen, and the presence of TPOAb might be correlated with severity of thyroid lymphocytic infiltration, despite the presence or absence of hypothyroidism [25]. Although genetic and environmental factors are known contributors of AITD, a causal 
relationship between obesity and thyroid autoimmunity has not been established, but observational data from the general population suggest that obesity may increase the risk of autoimmune diseases, such as rheumatoid arthritis and psoriatic arthritis [26, 27], probably due to the chronic proinflammatory state caused by the accumulation of VAT in obese patients. Compared with Caucasians, Chinese people are less obese and body fat distribution tends to accumulate in the abdominal cavity, which is more likely to form abdominal obesity. The overweight and obesity were significantly higher among male (41.2\% and 29.9\%) than among female (35.1\% and $28.4 \%$ ) participants in this study. Moreover, men had a higher prevalence of abdominal obesity than women (56.6\% in men and $47.6 \%$ in women). The prevalence of overweight, general obesity, and abdominal obesity in our study is higher than the data from China Health and Nutrition Survey (CHNS) [28]. However, the same as CHNS, the prevalence of overweight and general obesity is higher in men than women in our study. Meanwhile, there has been a sharp increase in the prevalence of abdominal obesity in men than in women in this study. Explanations for the higher prevalence of abdominal obesity in men than that in women might be due to the increased intake of beer by men and the different lifestyle, such as increased intake of high-fat diet and less exercise. Other explanations for the greater increase of abdominal obesity prevalence among men than women might be sex hormone responses to obesogenic environmental changes [29].

We also found worse lipid levels, such as had higher serum TG levels and lower HDL levels in men than in women in our study. Our data also showed that TPOAb titers was positively associated with TC, both in men and women. A similar finding was concluded that TPOAbpositive causes dyslipidemia in normal thyroid populations [30], while another study recorded no significant differences between TPOAb-positive and TPOAb-negative groups [31].

Results of the current study revealed that men with TPOAb (+) and/or TgAb (+) have a higher waist circumference than women. Notably, the waist circumference was positively associated with TPOAb in men but not in women, while hip circumference was positively associated with $\mathrm{TPOAb}$ in women but not in men. Our present findings also suggested that abdominal obesity is a risk factor for TPOAb positivity in men. Therefore, we consider that there is a gender difference in the relationship between obesity and HT in Chinese adults. The gender difference in the effects of sex hormones on HT between men and women may be due to the different roles of estradiol and testosterone in regulating immune responses $[32,33]$. Another possible explanation is that the gender differences in the associations of HT and obesity may be related to gender differences in the body fat distribution between men and women, which may lead to gender-specific changes in peripheral metabolism of thyroid hormones $[34,35]$. Obesity can lead to different and abnormal changes in sex hormones in men and women, which can lead to specific gender risks in thyroid autoimmune diseases. The gender association of obesity with thyroid autoimmune diseases may result from differences in adipokines between men and women [36]. Many adipokines, such as leptin and adiponectin, play an important role in regulating immunity and are considered to be a key link between obesity and obesity-related diseases. Therefore, the sex-specific effects of adipokines may thus be involved in the gender specificity of obesity with thyroid autoimmune diseases [37]. In obesity, it can directly or indirectly affect immune tolerance by altering the secretion of adipokines (mainly leptin, adiponectin, and mucin) and/or cytokines (interleukin-6, tumor necrosis factor alpha, and interleukin) [37]. The end result will be a shift from a Th2 to Th1 immune response, which is more likely to produce an autoimmune response. It is well established that $T$ helper cell 1 (Th1) type cytokines play pivotal roles in Hashimoto's thyroiditis and Graves' disease. Concordantly, cytokines that counteract Th1 responses, produced by Th2 cell subsets and $T$ regulatory cells, has been shown to have a protective effect on autoimmunity [38]. Our previous studies have found that an increased immune deviation of Th1 lymphocytes and compensatory accelerating activity of Treg cells in HT patients [39]. The visceral adipose tissue (VAT) includes macrophages, endothelial cells, and T cells with biased T cell receptors that may promote an immune response by producing an excess of proinflammatory cytokines [40]. In addition, VAT is a reservoir of regulatory T cells (Treg), and a small subset (5-15\%) of the T cell compartment is capable of controlling autoimmune responses. Researches showed that Treg cells are affected by leptin and involved in the control of autoimmunity [41] and thyroid cell apoptosis [42]. Another study suggests that leptin regulates the immune response of Thl [43]. The adipocytokines leptin and IL-6 inhibit regulatory T cells [26], whereas obesity alters cell-mediated Th1 immune responses, resulting in CD3 and CD4 T helper cells and CD8 T suppressor/cytotoxic cells defects [44, 45]. Some researchers have pointed out that obesity increases the risk of thyroid autoimmunity, which is related to leptin levels and other known predictors [13]. However, until now, the underlying mechanism of the association between abdominal obesity, especially visceral fat, and thyroid autoimmune diseases has not been clear.

Our results confirmed a relationship between obesity and AITD, especially abdominal obesity in men. Although women have a higher incidence of autoimmune diseases including AITD, the results of our research indicate that abdominal obesity is also a risk factor for TPOAb positivity in men. In terms of different ethnicities, race, and age, gender composition may have accounted for the difference in results obtained.

Our study has several strengths. First, only a few previous studies about the association of obesity, especially central obesity with thyroid autoimmunity in a large Chinese population, were available. We evaluated a relatively large sample of participants to examine the association of TPOAb and $\operatorname{TgAb}$ with obesity. Second, the data are highly reliable because all anthropometry and questionnaires are performed by the same trained research group with strict quality control, and all tests are performed under the same equipment and protocols. Third, we recruited community residents in Xinjiang China to make the results more representative. However, this study also has several limitations. 
First, because of the cross-sectional study, the direction of any causal relationship cannot be determined and the observed effect need to continue to verify in other people; thus, the prospective studies are needed. This study is the first part of our series of researches about the possible relationship between autoimmune thyroid disease and visceral adipose tissue and possible pathogenesis. Our subsequent follow-up study may make up for this limitation. Second, there is lack of a complete definition of chronic autoimmune thyroiditis. Third, other social and environmental variables, such as work stress, dietary habits, and sedentary lifestyles, which would have impact on obesity and thyroid autoimmunity, were not considered.

In conclusion, our present evaluation of the association of autoimmune thyroid disease with obesity, especially abdominal obesity, and dyslipidemia, has revealed that the prevalence of overweight, obesity, and abdominal obesity was higher in men. The waist circumference of men with Hashimoto's thyroiditis was higher than that of HT (-). The current study also showed that abdominal obesity can enhance the risk of thyroid autoimmunity. Further researches about the relationship between the abdominal obesity and thyroid diseases are warranted. Therefore, we suggest a follow-up study to understand the potential mechanism of visceral adipose tissue may contribute to promote susceptibility to thyroid autoimmunity.

\section{Data Availability}

The data used to support the findings of this study are available from the corresponding author upon request.

\section{Conflicts of Interest}

The authors declare no conflicts of interest.

\section{Authors' Contributions}

Xinling Wang and Yanying Guo designed the experiments, contributed to research guidance, and critically reviewed the manuscript. Yanrong Ma, Li Han, and Fuhui Ma provided intellectual inputs, performed the experiments, and contributed to the data acquisition. Yuyuan Zhang and Bei Xing contributed to the data collection. Jazyra Zynat and Suli Li Collated and analyzed the data and wrote the manuscript.

\section{Acknowledgments}

The authors thank all the participants in this survey. This study was supported by the National Natural Science Foundation of China (81560136) and Project in the people's Hospital of Xinjiang Uygur Autonomous region (20190105).

\section{Supplementary Materials}

Supplementary Table 1: clinical characteristics of the study participants. Supplementary Table 2: prevalence of Hashimoto's thyroiditis and obesity. Supplementary Table 3: characteristics of subjects in terms of the level of serum $\mathrm{TPOAb}$ and TgAb. Supplementary Table 4: correlation between metabolic and lipid parameters with serum TPOAb and $\mathrm{TgAb}$ levels. Supplementary Figure 1: flow diagram of participant enrollment in our study. Supplementary Figure 2: associations of TPOAb positivity with obesity, abdominal obesity, and hyperlipidaemia in men. (Supplementary Materials)

\section{References}

[1] R. Ajjan and A. Weetman, "The pathogenesis of Hashimoto's thyroiditis: further developments in our understanding," Hormone and Metabolic Research, vol. 47, no. 10, pp. 702-710, 2015.

[2] R. Ahmed, S. Al-Shaikh, and M. Akhtar, "Hashimoto thyroiditis," Advances in Anatomic Pathology, vol. 19, no. 3, pp. 181-186, 2012.

[3] K. Zaletel and S. Gaberscek, "Hashimotos thyroiditis: from genes to the disease," Current Genomics, vol. 12, no. 8, pp. 576-588, 2011.

[4] C. Selmer, J. B. Olesen, M. L. Hansen et al., "Subclinical and overt thyroid dysfunction and risk of all-cause mortality and cardiovascular events: a large population study," The Journal of Clinical Endocrinology \& Metabolism, vol. 99, no. 7, pp. 2372-2382, 2014.

[5] N. Yang, Z. Yao, Li Miao et al., "Novel clinical evidence of an association between homocysteine and insulin resistance in patients with hypothyroidism or subclinical hypothyroidism," Plos One, vol. 10, Article ID e0125922, 2015.

[6] M. Lu, C.-B. Yang, L. Gao, and J.-J. Zhao, "Mechanism of subclinical hypothyroidism accelerating endothelial dysfunction (review)," Experimental and Therapeutic Medicine, vol. 9, no. 1, pp. 3-10, 2015.

[7] O. Topaloglu, F. Gokay, K. Kucukler et al., "Is autoimmune thyroiditis a risk factor for early atherosclerosis in premenopausal women even if in euthyroid status?" Endocrine, vol. 44, no. 1, pp. 145-151, 2013.

[8] P. İşgüven, Y. Gündüz, and M. Kılıç, "Effects of thyroid autoimmunity on early atherosclerosis in euthyroid girls with Hashimoto's thyroiditis," Journal of Clinical Research in Pediatric Endocrinology, vol. 8, pp. 150-156, 2016.

[9] A. Afshin, A. Afshin, M. H. Forouzanfar et al., "Health effects of overweight and obesity in 195 countries over 25 years," The New England Journal of Medicine, vol. 377, pp. 13-27, 2017.

[10] M. Kivimäki, E. Kuosma, J. E. Ferrie et al., "Overweight, obesity, and risk of cardiometabolic multimorbidity: pooled analysis of individual-level data for 120813 adults from 16 cohort studies from the USA and Europe," The Lancet Public Health, vol. 2, no. 6, pp. e277-e285, 2017.

[11] A. B. Mitchell, J. W. Cole, P. F. McArdle et al., "Obesity increases risk of ischemic stroke in young adults," Stroke, vol. 46, no. 6, pp. 1690-1692, 2015.

[12] B. O. Asvold, T. Bjøro, and L. J. Vatten, "Association of serum TSH with high body mass differs between smokers and neversmokers," The Journal of Clinical Endocrinology and Metabolism, vol. 94, pp. 5023-5027, 2009.

[13] P. Marzullo, A. Minocci, M. A. Tagliaferri et al., "Investigations of thyroid hormones and antibodies in obesity: leptin levels are associated with thyroid autoimmunity independent of bioanthropometric, hormonal, and weight-related determinants," The Journal of Clinical Endocrinology \& Metabolism, vol. 95, no. 8, pp. 3965-3972, 2010.

[14] M. Rotondi, F. Magri, and L. Chiovato, "Thyroid and obesity: not a one-way interaction," The Journal of Clinical Endocrinology \& Metabolism, vol. 96, no. 2, pp. 344-346, 2011. 
[15] R. Thomas, "Obesity and thyroid function," Molecular \& Cellular Endocrinology, vol. 316, pp. 165-171, 2009.

[16] H. Khalili, A. N. Ananthakrishnan, G. G. Konijeti et al., "Measures of obesity and risk of Crohn's disease and ulcerative colitis," Inflammatory Bowel Diseases, vol. 21, no. 2, pp. 361-368, 2015.

[17] L. Ljung and S. Rantapää-Dahlqvist, "Abdominal obesity, gender and the risk of rheumatoid arthritis - a nested casecontrol study," Arthritis Research \& Therapy, vol. 18, p. 277, 2016.

[18] S. Meza-Perez and T. D. Randall, "Immunological functions of the omentum," Trends in Immunology, vol. 38, no. 7, pp. 526-536, 2017.

[19] The Society of Endocrinology, Chinese Medical Association, The Guideline for Diagnosis and Management of Thyroid Disease of China, The Society of Endocrinology, Chinese Medical Association, Beijing, China, 2008, http://www.cma. org.cn.

[20] Endocrinology Branch of Chinese Medical Association, "Expert consensus on comprehensive management of type 2 diabetes mellitus complicated with obesity in China," Chinese Journal of Endocrine Metabolism, vol. 32, no. 8, pp. 623-627, 2016.

[21] J. Zhu, R. Gao, S. Zhao et al., "Guidelines for the Prevention and treatment of dyslipidemia in Chinese adults," Chinese Circulation Journal, vol. 31, no. 10, 2016.

[22] X. Lian, "Iodine status and prevalence of thyroid disorders after introduction of mandatory universal salt iodization for 16 Years in China: a cross-sectional study in 10 cities," Thyroid Official Journal of the American Thyroid Association, vol. 26, p. 1125, 2016.

[23] China Health and Nutrition Survey (CHNS) (EB/OL), 2009, http://www.cpc.unc.edu/projects/china/data/datasets.

[24] Y. Tomer and T. F. Davies, "Searching for the autoimmune thyroid disease susceptibility genes: from gene mapping to gene function," Endocrine Reviews, vol. 24, no. 5, pp. 694-717, 2003.

[25] J. D. Mitchell, N. Kirkham, and D. Machin, "Focal lymphocytic thyroiditis in Southampton," The Journal of $\mathrm{Pa}$ thology, vol. 144, no. 4, pp. 269-273, 1984.

[26] L.-G. Hersoug and A. Linneberg, "The link between the epidemics of obesity and allergic diseases: does obesity induce decreased immune tolerance?" Allergy, vol. 62, no. 10, pp. 1205-1213, 2007.

[27] C. Procaccini, F. Carbone, M. Galgani et al., "Obesity and susceptibility to autoimmune diseases," Expert Review of Clinical Immunology, vol. 7, no. 3, pp. 287-294, 2011.

[28] B. Xi, Y. Liang, T. He et al., "Secular trends in the prevalence of general and abdominal obesity among Chinese adults, 1993-2009," Obesity Reviews, vol. 13, no. 3, pp. 287-296, 2012.

[29] S. Yoo, H.-J. Cho, and Y.-H. Khang, "General and abdominal obesity in South Korea, 1998-2007: gender and socioeconomic differences," Preventive Medicine, vol. 51, no. 6, pp. 460-465, 2010.

[30] D. Kang, Q. Yin, X. Yan et al., "Serum cholesterol levels in middle-aged euthyroid subjects with positive thyroid peroxidase antibodies," International Journal of Clinical and Experimental Medicine, vol. 8, pp. 21623-21628, 2015.

[31] B. J. Wells and W. J. Hueston, "Are thyroid peroxidase antibodies associated with cardiovascular disease risk in patients with subclinical hypothyroidism?" Clinical Endocrinology, vol. 62, no. 5, pp. 580-584, 2005.
[32] Y. Chen, Y. Chen, F. Xia et al., "A higher ratio of estradiol to testosterone is associated with autoimmune thyroid disease in males," Thyroid, vol. 27, no. 7, pp. 960-966, 2017.

[33] P. Habib, D. Dreymueller, B. Rösing et al., "Estrogen serum concentration affects blood immune cell composition and polarization in human females under controlled ovarian stimulation," The Journal of Steroid Biochemistry and Molecular Biology, vol. 178, pp. 340-347, 2018.

[34] F. Santini, P. Marzullo, M. Rotondi et al., "Mechanisms in endocrinology: the crosstalk between thyroid gland and adipose tissue: signal integration in health and disease," European Journal of Endocrinology, vol. 171, no. 4, pp. R137-R152, 2014.

[35] L. Fontenelle, M. Feitosa, J. Severo et al., "Thyroid function in human obesity: underlying mechanisms," Hormone and Metabolic Research, vol. 48, no. 12, pp. 787-794, 2016.

[36] M. Aguado, D. Martínez-Urbistondo, A. Solomon, and J. A. Martínez, "Gender-dependent differences in the responses of human adipocytes to leptin lipolytic action," Methods and Findings in Experimental and Clinical Pharmacology, vol. 30, no. 1, pp. 7-11, 2008.

[37] M. Versini, P.-Y. Jeandel, E. Rosenthal, and Y. Shoenfeld, "Obesity in autoimmune diseases: not a passive bystander," Autoimmunity Reviews, vol. 13, no. 9, pp. 981-1000, 2014.

[38] T. R. Mosmann and S. Sad, "The expanding universe of T-cell subsets: Th1, Th2 and more," Immunology Today, vol. 17, no. 3, pp. 138-146, 1996.

[39] Y. Guo, J. Zynat, S. Xing et al., "Immunological changes of T helper cells in flow cytometer-sorted CD4 T cells from patients with Hashimoto's thyroiditis," Experimental and Therapeutic Medicine, vol. 15, pp. 3596-3602, 2018.

[40] Y. H. Yang, B. Youm, A. Vandanmagsar et al., "Obesity increases the production of proinflammatory mediators from adipose tissue $\mathrm{T}$ cells and compromises TCR repertoire diversity: implications for systemic inflammation and insulin resistance," Journal of Immunology, vol. 185, pp. 1836-1845, 2010.

[41] S. Sakaguchi, M. Ono, R. Setoguchi et al., "Foxp3CD25CD4 natural regulatory $\mathrm{T}$ cells in dominant self-tolerance and autoimmune disease," Immunological Reviews, vol. 212, pp. 8-27, 2010.

[42] S. H. Wang, G.-H. Chen, Y. Fan, M. Van Antwerp, and J. R. Baker, "Tumor necrosis factor-related apoptosis-inducing ligand inhibits experimental autoimmune thyroiditis by the expansion of CD4+CD25+Regulatory T cells," Endocrinology, vol. 150, no. 4, pp. 2000-2007, 2009.

[43] G. Matarese, E. H. Leiter, and A. La Cava, "Leptin in autoimmunity: many questions, some answers," Tissue Antigens, vol. 70, no. 2, pp. 87-95, 2007.

[44] S. Tanaka, F. Isoda, Y. Ishihara, M. Kimura, and T. Yamakawa, "T lymphopaenia in relation to body mass index and TNFalpha in human obesity: adequate weight reduction can be corrective," Clinical Endocrinology, vol. 54, pp. 347-354, 2010.

[45] H. Yang, Y.-H. Youm, B. Vandanmagsar et al., "Obesity accelerates thymic aging," Blood, vol. 114, no. 18, pp. 3803-3812, 2009. 